\title{
Patent arterial duct, bottle-meal, and fatal myocardial ischaemia*
}

\author{
Simon Fluri, ${ }^{1}$ Mladen Pavlovic, ${ }^{2}$ Bendicht P. Wagner ${ }^{1}$ \\ ${ }^{1}$ Department of Pediatrics, Pediatric Intensive Care Unit, University Hospital; ${ }^{2}$ Pediatric Cardiology, Inselspital, \\ Bern, Switzerland
}

\begin{abstract}
A patent arterial duct in pre-term neonates is frequent. Systemic complications consecutive to leftto-right shunting are well known but fatal myocardial ischaemia has not been described till now. The presented premature baby died from catecholamine refractory cardiogenic shock. Autoptic examination revealed acute ischaemic changes predominantly in the inner third of myocardium, speaking of coronary hypoperfusion due to a steal phenomenon secondary to the patent arterial duct.
\end{abstract}

Keywords: Premature newborn; steal phenomenon; myocardial infarction; oral-feeding route; prevention

Received: 4 May 2009; Accepted: 22 November 2009; First published online 1 March 2010

A PATENT ARTERIAL DUCT IS A FREQUENT FINDING in premature infants. Substantial left-to-right shunting through the ductus is associated with several systemic complications: the risk of intraventricular haemorrhage, necrotising enterocolitis, decreased renal function, bronchopulmonary dysplasia, and death may be increased. ${ }^{1}$ However, fatal myocardial ischaemia does not appear as a known complication of the patent arterial duct.

\section{Case report}

This 1110-gram twin boy was delivered by cesarean section at 27 1/7 weeks of gestation. Apgar scores were 1,3 , and 5 at 1,5 , and 10 minutes, respectively. He was intubated in the delivery room and a surfactant was administered twice. The chest $\mathrm{X}$-ray showed grade IV hyaline membrane disease. On day 5 of life the boy was extubated and put on nasal continuous positive airway pressure therapy for another 23 days. Apnea of pre-maturity was successfully treated with caffeine citrate; no additional

*This case report obtained the approval for publication from the Institutional Review Board.

Correspondence to: Dr Simon Fluri, MD, Department of Pediatrics, Pediatric Intensive Care Unit, Inselspital, CH-3010 Bern. Tel: +41 316322 111; Fax: +41316320 784; E-mail: simon.fluri@insel.ch oxygen was needed. At 1 month of life, cardiac auscultation showed a 4/6 systolic murmur. A patent arterial duct of 2-3 millimetres diameter with a relevant left-to-right shunt was confirmed in echocardiography. No signs of cardiovascular decompensation was found: renal function with creatinine at 48 micrograms per litre and digestion were normal; respiration was stable, that is respiration rate at 60 per minute and $95 \%$ oxygen saturation, chest $\mathrm{X}$-ray showed mild cardiomegaly and hypercirculation; systolic/diastolic/mean blood pressure was 50/28/36 millimetres of mercury, heart rate at 150 per minute. Cerebral sonography showed an elevated resistance index (0.98) with decreased intracerebral diastolic flow velocity. ${ }^{2}$ No attempt to close patent arterial duct was initiated.

In the sixth week of life, at the gestational age of $326 / 7$ weeks, the boy was offered to drink from the bottle. He is on an eight-meal regimen with 30 millilitres of human milk per meal, that is 150 millilitres per kilogram per day. After 45 minutes of the bottle, he started abruptly with tachypnea, retractions, flaring of the nasal alae, grunting, and oxygen requirement of up to a $30 \%$. Oscillometrically measured blood pressure was 40/22/26 millimetres of mercury. Respiratory distress was followed by bradypnea and clinical signs of shock. 
Mainly metabolic acidosis $\left(\mathrm{pH}\right.$ 6.7, $\mathrm{CO}_{2} 58$ millimetres of mercury, base excess 26.9 millimols per litre, bicarbonate 7.2 millimols per litre) and arterial hypotension (34/28/30 millimetres of mercury) were found. He was intubated with ketamine and pancuronium and ventilated with a $90 \%$ oxygen requirement and a peak inspiratory pressure going up to a maximum of 26 centimetres in the water column to obtain tidal volumes of 7.5 millilitres per kilogram. Echocardiography was performed showing an important impairment of left ventricular function with an ejection fraction of $19 \%$.

Blood pressure was 35/28/30 millimetres of mercury. Arterial and central venous catheters were placed and first dobutamine and second epinephrine were administered to maintain blood pressure in a low normal range, that is, 34/24/29 millimetres of mercury. In the absence of dehydration and the high suspicion of cardiogenic shock, no fluids were given. Troponin I was 46.9 micrograms per litre. No electrocardiography was performed. One hour before death, severe bradycardia and hypotension (24/12/ 18 millimetres of mercury) finally required heart massage and epinephrine boli. The boy died 6 hours after the onset of respiratory distress from irreversible, catecholamine refractory cardiogenic shock.

The autopsy showed a patent arterial duct. The coronary arteries were inconspicuous. The microscopic examination of the heart revealed acute ischaemic changes of the inner third of the myocardium, including subendocardial bands of necrosis (Fig 1a) as well as multiple foci of wavy myocardial fibres (Fig 1b) in both ventricles. No myocarditis was present. No signs of pulmonary aspiration were found microscopically.

\section{Discussion}

Fatal myocardial ischaemia in the newborn is extremely rare. ${ }^{3}$ Most often it is seen in association with congenital cardiac disease, specifically, obstructive lesions, that is, aortic stenosis, anomalous origin of left coronary artery, and tricuspidal abnormalities. Another cause of neonatal myocardial infarction is embolic injuries of coronary arteries. Coronary thrombosis with focal intimal thickening and coronary spasm in stressed newborns is reported. Impaired coronary perfusion because of increased work demands created by unusually brisk pulmonary vasoconstriction in newborn with pulmonary hypoxia is described ${ }^{4}$. In contrast, ductal steal phenomenon secondary to patent arterial duct has not been described so far as a cause of fatal myocardial ischaemia.

A recent transthoracic Doppler echocardiography study showed decreased left anterior descending
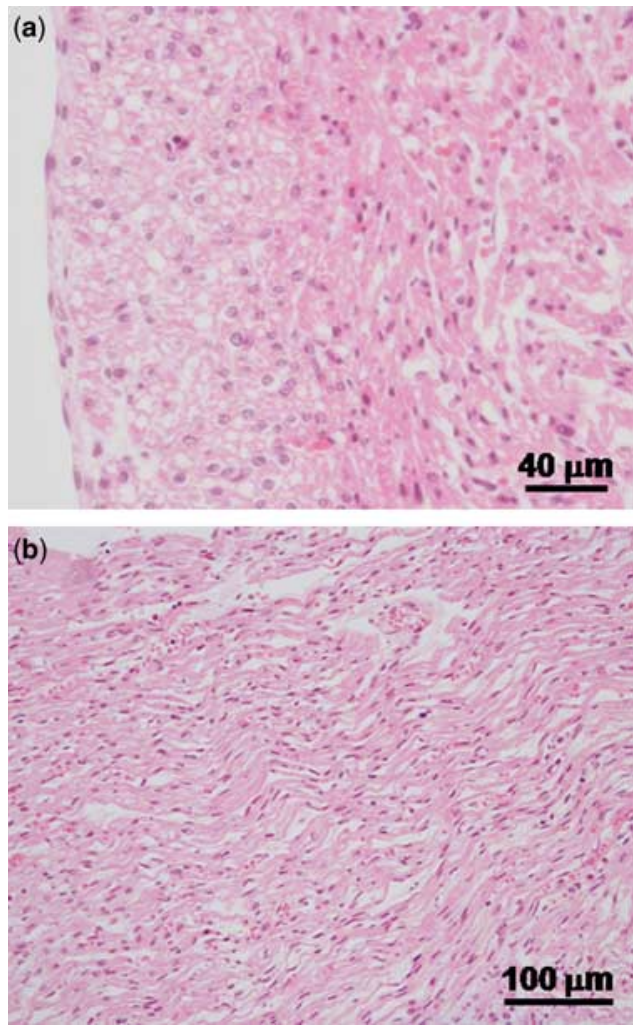

Figure 1.

Postmortem histopathologic examination of the heart yielding acute ischaemic changes; haematoxylin and eosin-stained tissue sections. (a) Subendocardial band of myocyte necrosis (asterisks) with bypereosinophilia and pycnotic nuclei and (b) wavy myocard fibres.

coronary artery blood flow as a result of patent arterial duct; coronary perfusion improved after coil closure of arterial duct. ${ }^{5}$ In another study, ST depressions on electrocardiogram suggesting ischaemia were found in a group of neonates with patent arterial duct and respiratory distress syndrome; ST depressions disappeared after surgical ligation of the arterial duct. ${ }^{6}$

In our case, there was no pulmonary impairment, metabolic disturbance, or infectious disease. Respiratory distress syndrome was the first sign of cardiac decompensation without any sign of bronchopulmonary aspiration. Blood pressure could be maintained within low normal ranges with the exception of the last 2 hours of life. The fact that acute ischaemic changes were predominantly found in the inner layer of the myocardium supports the diagnosis of myocardial ischaemia as the result of the ductal steal phenomenon secondary to a patent arterial duct, because the inner third of the myocardium is typically perfused during diastole. The ductal steal phenomenon results in a reduced diastolic blood pressure and therefore compromises coronary artery perfusion, comparable to the well-known and routinely measured 
reduced diastolic middle cerebral artery blood flow velocity secondary to patent arterial duct. ${ }^{7}$

Bottle feeding represents an important physical effort in every neonate. Very low birthweight newborns cannot increase their resting metabolism by more than $15 \%$ for physical activity. ${ }^{8}$ Bottle feeding significantly increases respiratory rate and can decrease oxygen saturation. 'This physical activity increases cardiac output and therefore myocardial oxygen consumption. With regard to these facts, the oral feeding route may have been the trigger of this abrupt decompensation.

\section{Conclusion}

Newborns with a patent arterial duct and extremely low diastolic blood pressure may be at increased risk for myocardial infarction secondary to a coronary steal phenomenon even in the absence of other organ manifestations. A cautious and slow enteral feeding regimen with extended feeding by gastric tubes may be of benefit, comparable to other neonates with a cardiovascular compromise. Early diagnosis and interventions to increase diastolic pressure and therefore coronary artery perfusion will contribute to improve the survival of premature children with patent arterial duct.

\section{References}

1. Van Overmeire B, Smets K, Lecoutere D, et al. A comparison of ibuprofen and indomethacin for closure of patent ductus arteriosus. N Engl J Med 2000; 343: 674-681.

2. Couture A, Veyrac C, Baud C, Saguintaah M, Ferran JL. Advanced cranial ultrasound: transfontanellar Doppler imaging in neonates. Eur Radiol 2001; 12: 2399-2410.

3. Kilbride H, Way GL, Merenstein GB, Winfield JM. Myocardial infarction in the neonate with normal heart and coronary arteries. Am J Dis Child 1980; 134: 759-762.

4. Rowe RD, Hoffmann T. Transient myocardial ischemia of the newborn infant: a form of severe cardiorespiratory distress in fullterm infants. J Pediatr 1972; 81: 243.

5. Harada K, Toyono M, Tamura M. Effects of coil closure of patent ductus arteriosus on left anterior descending coronary artery blood flow using transthoracic Doppler echocardiography. J Am Soc Echocardiogr 2004; 17: 659-663.

6. Way GL, Pierce JR, Wolfe RR, McGrath R, Wiggins J, Merenstein GB. ST depression suggesting ischemia in neonates with respiratory distress syndrome and patent ductus arteriosus. J Pediatr 1979; 95: 609-610.

7. Weir FJ, Ohlsson A, Myhr TL, Fong K, Ryan ML. A patent ductus arteriosus is associated with reduced middle cerebral artery blood flow velocity. Eur J Pediatr 1999; 158: 484-487.

8. Polin, Fox. Fetal and Neonatal Physiology, 2nd edition. WB Saunders Company, Philadelphia, USA, 1998.

9. Blaymore Bier JA, Ferguson AE, Morales Y, Liebling JA, Oh W, Vohr BR. Breastfeeding infants who were extremely low birth weight. Pediatrics 1997; 100: E3. 